\title{
On Blade Arrangement for Multi-Blade Rotors
}

\author{
K.L. Hitz' ${ }^{1}$ and D.H. Wood ${ }^{1,2, *}$ \\ 'School of Engineering, University of Newcastle, NSW 2308, Australia \\ ${ }^{2}$ current address: Schulich School of Engineering, University of Calgary \\ (dhwood@ucalgary.ca)
}

\begin{abstract}
This paper concerns the blades of multi-bladed water-pumping windmills when they have variable mass and centre of mass. The paper explores blade arrangement strategies that will minimize the eccentricity of the rotor centre of mass and hence any rotor-induced vibration. The number of blades in the rotor is assumed to equal the number made in each production batch, in contrast to the case where a batch of up to 22 blades was optimally matched to produce two- and three-bladed rotors, Hitz \& Wood [1]. Using the measured mass and centre of mass of 24 blades for the rotor of a $26 \mathrm{ft}$ Kijito windmill described by Harries [3], three strategies are considered. Random matching of the blades is shown to become increasingly effective as blade number increases. Pairing the blades by ordering in the product of mass and centre of mass, $d$, followed by random selection of pairs also produces rotors with low eccentricities. The numerical experiments show that the best strategy involving random selection is to pair by ordering, swapping the blades of every second pair, and then randomly arrange the resulting pairs. Finally, a heuristic based on blade pairing is shown to give eccentricities which are high compared to the minimum value determined exactly for 12 blades or less, but apparently low enough to be useful.
\end{abstract}

Keywords: windmill blades, multi-bladed rotor, matching, branch and bound algorithm, blade arrangement.

\section{INTRODUCTION}

Hitz and Wood [1] studied the problem of forming wind turbine rotors by optimally matching pairs or triples of blades without recourse to additional actions such as increasing the mass of the lightest blade. It was assumed that the blades were made in large batches. Matching was considered optimal when it minimised the sum of the squared differences in the chosen matching parameter for all blade pairs or triples in a batch. Ref.[1] considered both simple and complex matching. It was proved that a parameter such as blade mass, or centre of mass, CoM, allows optimal matching simply by ordering in terms of that parameter. The only matching parameter that is mentioned in the IEC standard for small wind turbine safety, Ref. [2], is the eccentricity of the rotor CoM, e. Using this parameter leads to complex matching as the contribution to $e$ from one blade depends on that of at least one other blade. Then ordering does not necessarily produce the optimal matching but, in all cases considered in Ref. [1], it came very close. A branch and bound algorithm was developed for complex matching and was shown to determine the optimal matching in a realistic time on a laptop PC for batches of at least 20 blades. Computational time rose rapidly with increasing batch size and it would be 


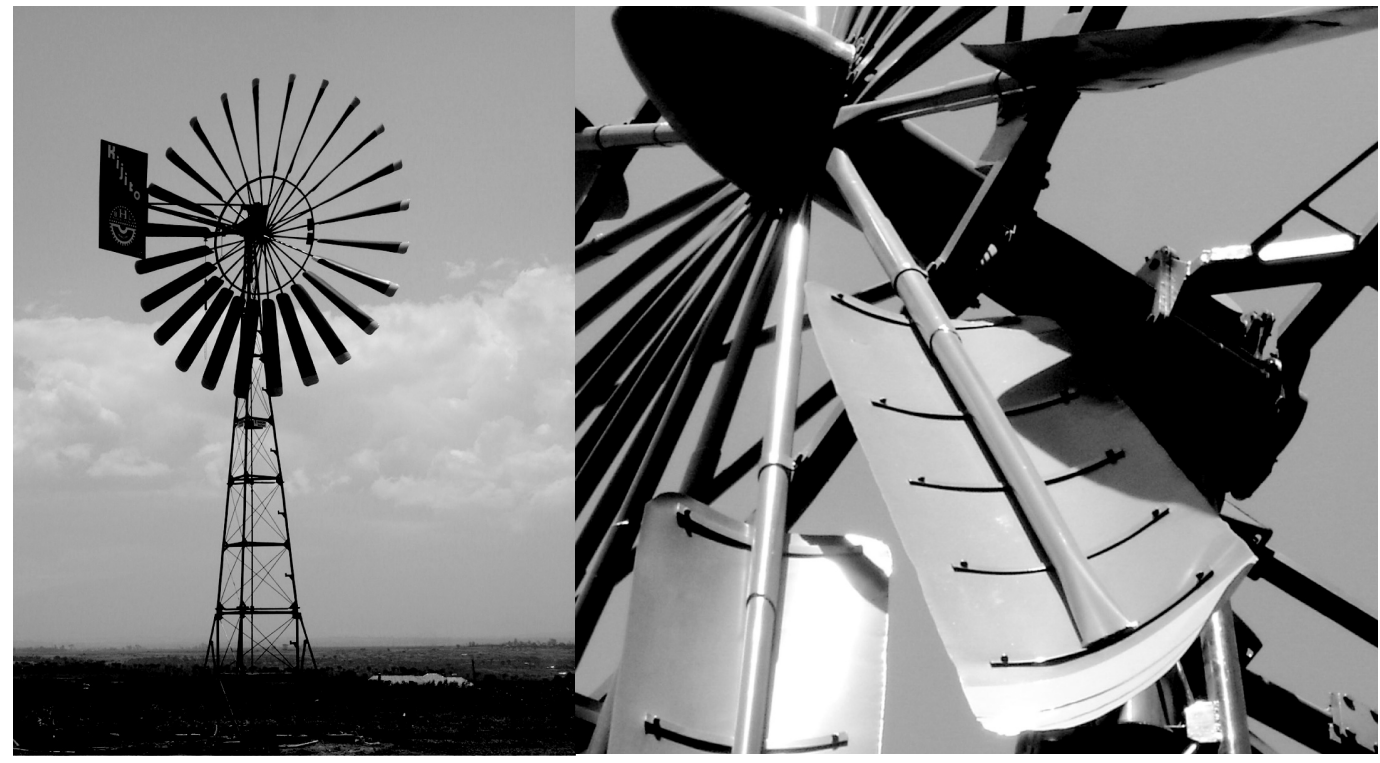

Figure 1: The Kijito $26 \mathrm{ft}$, 24-bladed water pumping windmill. The view on the right shows the method of blade construction.

impossible to obtain optimal matching from batches larger than about 22 in realistic time even with extensive computational resources.

Ref. [1] determined the optimal pairing of an actual batch of 8 blades, and then randomly generated larger batches for exploratory purposes. The measured blade mass and CoM were nearly independent and independence was assumed in the computer studies of larger batches. Variations in the mass and CoM of $1 \%$ (approximately that in the actual batch of 8 ) and then $5 \%$ were investigated.

Two and three-bladed rotors are the most common for electricity-generating wind turbines but represent a special case for matching in that the arrangement of the blades within the rotor has no effect on the value of the matching parameter. Thus swapping the second and third blades on a three-bladed rotor, for example, does not change $e$. It is easy to show that optimal matching for blade number, $N,=4$ is equivalent to finding two optimal pairs which must be arranged at right angles; altering this arrangement will increase $e$. Optimal matching for $N=6$, however, cannot be interpreted in terms of three pairs or two triples and the intermediate case of $N=5$, bears no relation to selecting pairs or triples. In other words, blade matching for multibladed rotors with $N>3$, must consider the arrangement of the blades in the rotor.

In this paper we consider optimal matching for $N>3$, with emphasis on a practical example that is close to the opposite of those considered in Ref. [1]. This is the water pumping windmill produced by Kijito Windpower Ltd in Kenya, Harries [3]. The windmill, shown in Figure 1, has 24 blades made as required in batches of the same number ${ }^{1}$. For this large $N$, a suitable strategy for arranging the blades, or the demonstration that random selection is adequate, is much preferable to modifying individual blades by adding or removing mass.

\section{BLADE DATA}

The mass and CoM of 24 blades were measured before the construction of a rotor for a $26 \mathrm{ft}$ windmill at the Kijito factory. The values for each blade are given in Table 1 and displayed in 
Table I: Blade mass and centre of mass for a Kijito $26 \mathrm{ft}$, 24-bladed water pumping windmill

$\begin{array}{ccc}\text { Blade Number } & \text { Blade Mass }(\mathbf{k g}) & \text { Centre of Mass }(\mathbf{m}) \\ 1 & 11.3 & 1.62 \\ 2 & 10.9 & 1.6 \\ 3 & 11.3 & 1.62 \\ 4 & 10.9 & 1.615 \\ 5 & 11.15 & 1.628 \\ 6 & 11.05 & 1.598 \\ 7 & 10.8 & 1.602 \\ 8 & 11.2 & 1.596 \\ 9 & 10.9 & 1.595 \\ 10 & 11.15 & 1.618 \\ 11 & 11.35 & 1.625 \\ 12 & 11.1 & 1.624 \\ 13 & 10.7 & 1.603 \\ 14 & 11.25 & 1.6 \\ 15 & 11.25 & 1.617 \\ 16 & 11.1 & 1.621 \\ 17 & 10.85 & 1.609 \\ 18 & 11.25 & 1.62 \\ 19 & 10.9 & 1.594 \\ 20 & 11.3 & 1.609 \\ 21 & 10.8 & 1.592 \\ 22 & 11.05 & 1.618 \\ 23 & 11.1 & 1.621 \\ 24 & 11.1 & 1.622 \\ & & \end{array}$

Figure 2. Note that the blade number in the first column is arbitrary and indicates only the order in which the blades were measured.

In contrast to the independence seen in the batch of 8 composite blades considered in Ref. [1], the correlation coefficient between blade mass and CoM in Table 1 is 0.550 . This high value

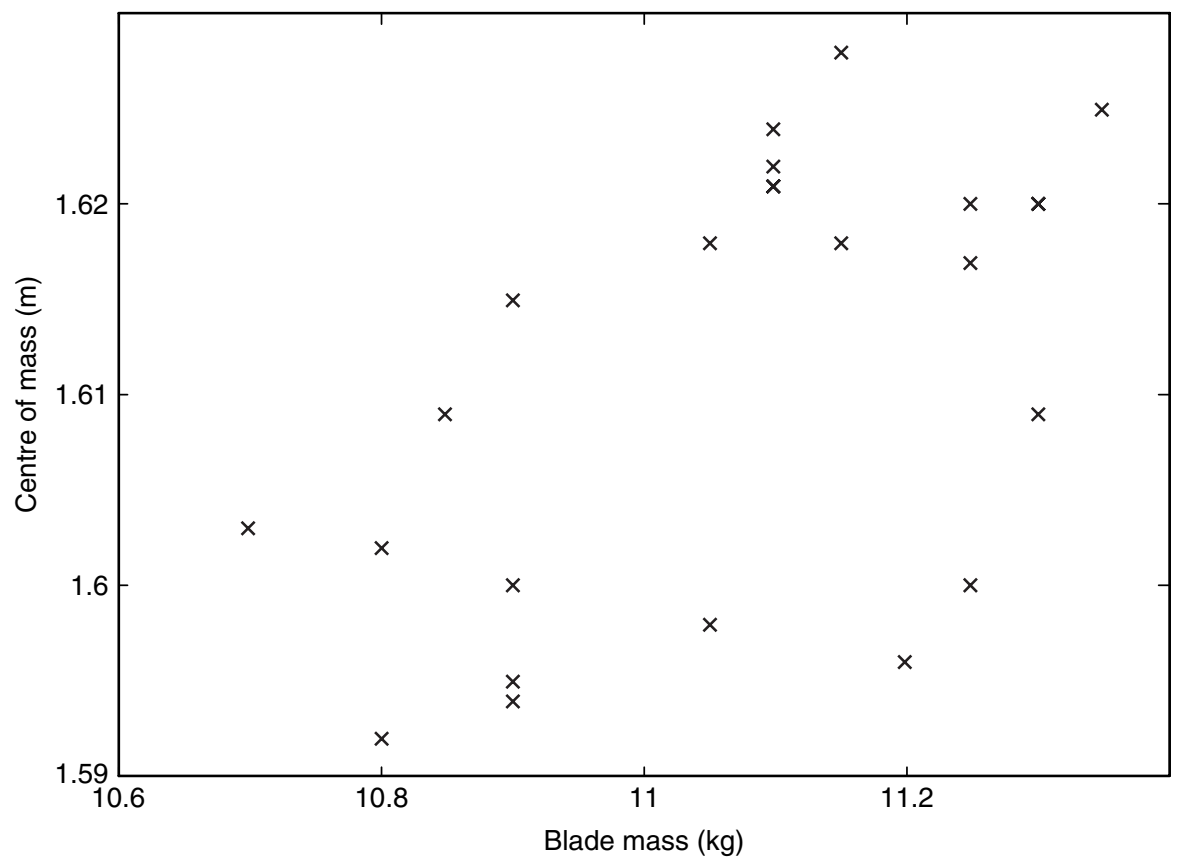

Figure 2: Mass and centre of mass from Table 1. 
probably arises from variations in the length of the circular spars shown clearly in the right hand part of Figure 1. These variations would cause changes of the same sign to the mass and CoM. The r.m.s. variations in blade mass and $\mathrm{CoM}$ are $1.7 \%$ and $0.7 \%$ respectively.

\section{BLADE MATCHING AND ARRANGING}

We consider only matching and arranging to minimise $e$. It is assumed that the variability occurs only in $d_{l}$, the product of the blade mass, $m_{l}$, and CoM, $r_{l}$, of blade $l$, and not in the setting angles of the blades. Furthermore, all $d_{l}$ are assumed to lie in the plane of rotation. Using Cartesian co-ordinates to describe the locations of $d_{l}$, with the $x$-axis arbitrarily aligned with the axis of blade $N$, the vector sum of $d$ for the rotor can be written as

$$
\mathbf{d}=\sum_{1}^{N} d_{l} \cos (2 \pi l / N) \mathbf{i}+\sum_{1}^{N} d_{l} \sin (2 \pi l / N) \mathbf{j}
$$

where $\mathrm{i}$ and $\mathrm{j}$ are the unit vectors on the $x$-and $y$-axes respectively. The eccentricity, $e$, is given by

$$
\begin{gathered}
e=\sqrt{d^{2}} / \sum_{1}^{N} m_{l} \quad \text { where } d^{2}=\sum_{1}^{N} \sum_{1}^{N} a_{m l} d_{m} d_{l} \\
\text { and } \quad a_{m l}=\cos (2 \pi(l-m) / N)
\end{gathered}
$$

Note that $a_{m l}=a_{l m}$ and $a_{m m}=1$ for all $l$ and $m$. The fundamental difference between matching for small $N$ when compared to matching and arrangement for larger $N$ can be seen in terms of $a_{l m}$ considered as an element of the matrix of coefficients, $\boldsymbol{a}$. For $N=2, a_{12}=a_{21}=-1$ and for $N=3$ all the off-diagonal elements are equal to $-1 / 2$. Thus the arrangement of blades in either case does not affect the eccentricity. For $N=4$, the off-diagonal elements are either -1 or 0 , so swapping within the two pairs of blades, say $(1,3)$ and $(2,4)$, does not change $e$ but any other swap will. For $N>4$, the coefficients change sign, so the blade arrangement is critical.

If the number of blades in the batch is $N$, the arrangement that minimises the $e$ also minimises

$$
\sum_{m=1+1 l=1}^{N} \sum_{l m}^{N} d_{l} d_{m}
$$

and is an example of complex matching.

Note that (2) is independent of the co-ordinate system used in (1). Now define $d_{l}=D+\delta_{l}$, where $D$ is the mean for $N$ blades and $\delta$ is the deviation. The sum of the off-diagonal elements of $\boldsymbol{a}$ is $-N$ and the obvious sum of the diagonal elements is $N$. This removes $D$ from the equation for the eccentricity to leave

$$
d^{2}=\sum_{1}^{N} \delta_{l}^{2}+\sum_{m=1+1 l=1}^{N} \sum_{l m}^{N} 2 a_{l m} \delta_{l} \delta_{m}
$$

The absence of $D$ in (4) demonstrates the obvious point that a non-zero eccentricity can arise only from deviations from the average value.

In principle, exact algorithms, such as the one mentioned in Section 5, can determine the optimal blade arrangement that would minimise (3) but in the present case there are $N$ ! possible arrangements of the blades. Since $24 ! \approx 6.2045 \times 10^{23}$, the time required would be excessive, based on the numerical experiments described in Ref. [1] of which the largest had $3.6212 \times 10^{10}$ possible matchings. We were able to exactly determine the optimal blade 
arrangement only for rotors with up to 12 blades. For larger $N$, there are at least three possible ways forward:

1. investigate the usefulness of random blade selection, justified by the arguments given at the start of the next Section,

2. simply order the blades into pairs or triples, and then attempt to find the best of the significantly reduced number of possible arrangements, or

3. develop a heuristic optimisation method that uses much less c.p.u. time to approximate the optimum arrangement.

The remainder of this paper explores all three options. For $N=24$, the number of possible arrangements under option 2 is $12 ! \times 2^{12}=1.962 \times 10^{12}$ which is a significant reduction of the full problem but still greater than the largest number of possible matchings considered in Ref. [1]. Moreover, the individual terms in the summand in the second sum of Equation (4) can be positive or negative, whereas all the corresponding terms for the cases of $N=2$ and 3 considered in [1] were positive. This means that the branch and bound strategy of Ref. [1] which minimises c.p.u. time by discarding "partial lists" of blades that have already exceeded the current minimum sum, cannot be used here. It was found that c.p.u. time depended strongly on the maximum number of "complete lists" searched in the numerical experiments of Ref.[1]; the maximum number was 95,187.

\section{RANDOM BLADE ARRANGING}

It seems obvious that random selection will eventually become a good strategy as $N$ increases. To investigate this, random selections for a 12-bladed rotor were made from the first 12 blades listed in Table 1 and then for a 24-bladed rotor using all the blades. The r.m.s. deviation in $d$ was 0.3932 for the first 12 blades and 0.3879 for the complete set. 10,000 random selections were made for each $N$ and the resulting histograms of 20 bins are shown in Figure 3. It is clear that

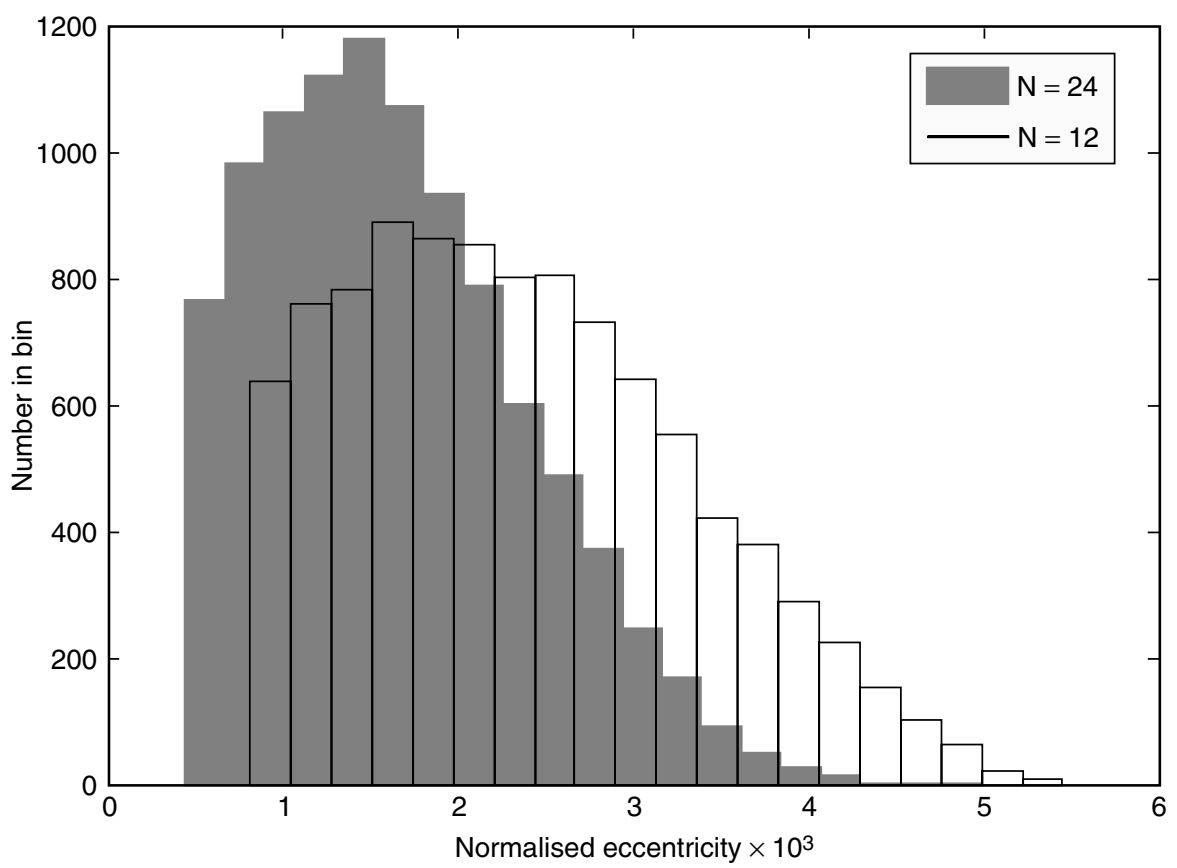

Figure 3: Histograms for random selection of 12- and 24-blade rotors from the data in Table 1. The eccentricity is normalised by the rotor mass and blade radius. 
increasing $N$ improves random selection as a strategy, as all 10,000 selections for $N=24$ resulted in $e<0.005 R$, the default value in Ref. [2]. The c.p.u. time required for the Matlab program to make 10,000 random selections for $N=24$ was always less than 14.5 seconds on an Intel Centrino Duo laptop.

\section{BLADE ARRANGEMENT BASED ON PAIRING}

Pairing by ordering is easy, and the equation for the eccentricity simplifies if the $N$ blades are considered as $N / 2$ pairs, provided that $N$ is even. A similar simplification does not appear possible if the blades are first ordered as triples.

Consider typical pairings $(l, l+N / 2)$ and $(m, m+N / 2)$. Since $\cos (x+\pi)=-\cos x$ it is easy to show that (4) can be rewritten as

$$
d^{2}=\sum_{1}^{N} \delta_{l}^{2}-\sum_{l=1}^{N} \delta_{l} \delta_{l+N / 2}+\sum_{m=l+1 l=1}^{N} \sum_{l m}^{N} a_{l}\left(\delta_{l}-\delta_{l+N / 2}\right)\left(\delta_{m}-\delta_{m+N / 2}\right)
$$

where only the last (double) sum requires minimisation and only this sum will be referred to again in this section. Recall the convention from Ref. [1] that $\delta_{l+N / 2}>\delta_{l}$ for all pairs so that the summand has the sign of $a_{l m}$. It may be beneficial to swap blades in some of the pairs in order to change the sign of $\left(\delta_{l}-\delta_{l+N / 2}\right)\left(\delta_{m}-\delta_{m+N / 2}\right)$.

The 24 blades in Table 1 were ordered to determine pairs and these pairs were randomly arranged to form the rotor. The histogram from 10,000 blade arrangements in Figure 4 labelled "without swapping" can be compared to Figure 3: pairing significantly reduces the mean eccentricity. If every second blade pair is swapped before random arranging, the histogram labelled "with swapping" shows a further significant decrease in the mean eccentricity from 0.00065 to 0.00024 . Table 2 lists the blade arrangement with $e=1.20 \times 10^{-6} R$ which is the smallest value from the numerical experiment.

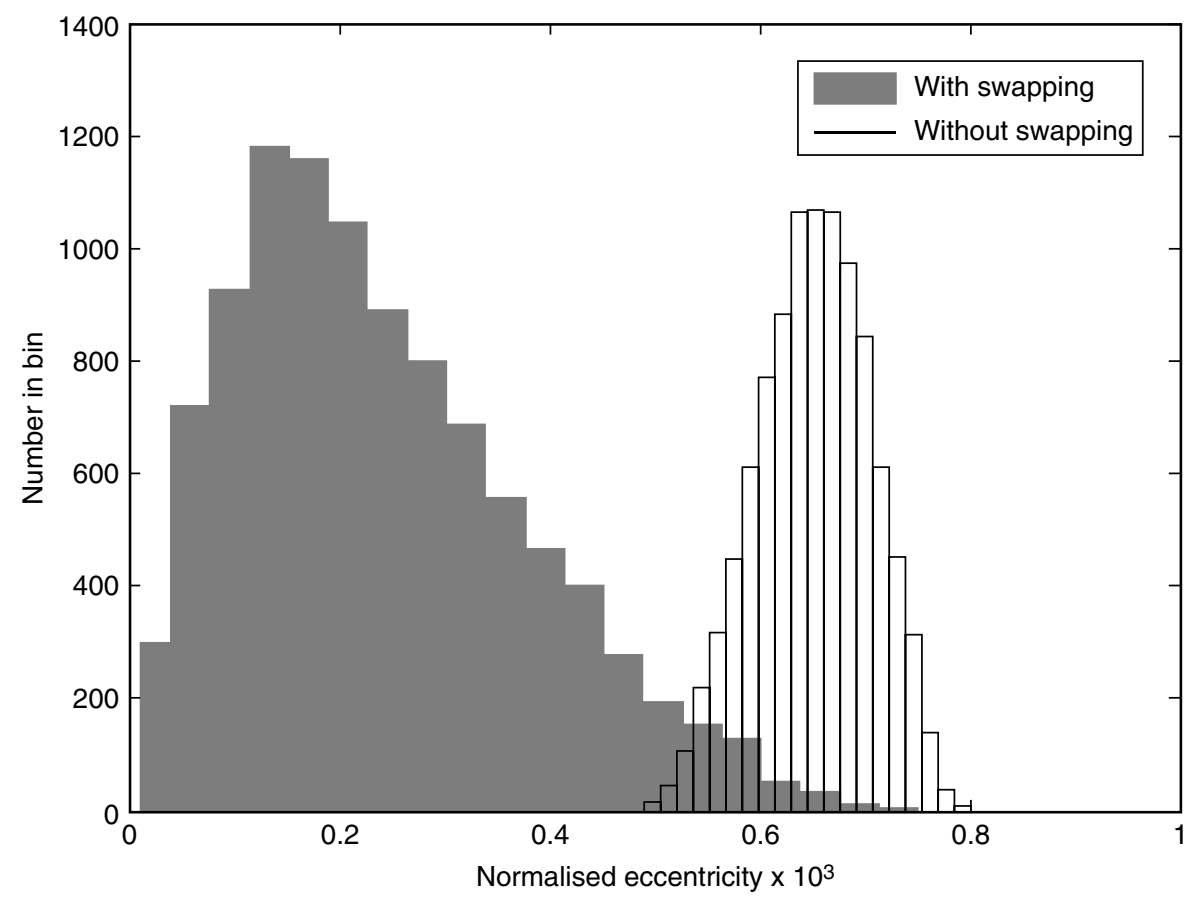

Figure 4: Histograms for random selection of 12 pairs for 24 -blade rotors from the data in Table 1. 
Table 2: Blade arrangement for normalised eccentricity $=1.20 \times 10^{-6}$

\begin{tabular}{|c|c|c|c|}
\hline Blade Number, $I$ & Blade Number from Table 1 & $D+\delta_{l}(\mathbf{k g m})$ & $\delta_{l}-\delta_{l+N / 2}(\mathrm{kgm})$ \\
\hline 1 & 13 & 17.1521 & -0.0415 \\
\hline 2 & 9 & 17.3855 & -0.0545 \\
\hline 3 & 23 & 17.9931 & 0.1142 \\
\hline 4 & 19 & 17.3746 & 0.0730 \\
\hline 5 & 16 & 17.9931 & -0.0069 \\
\hline 6 & 6 & 17.6579 & -0.2173 \\
\hline 7 & 4 & 17.6035 & 0.1458 \\
\hline 8 & 15 & 18.1913 & 0.0096 \\
\hline 9 & 10 & 18.0407 & -0.1115 \\
\hline 10 & 11 & 18.4437 & 0.1377 \\
\hline 11 & 18 & 18.2250 & -0.0810 \\
\hline 12 & 12 & 18.0264 & 0.0222 \\
\hline 13 & 21 & 17.1936 & \\
\hline 14 & 2 & 17.4400 & \\
\hline 15 & 22 & 17.8789 & \\
\hline 16 & 7 & 17.3016 & \\
\hline 17 & 14 & 18.0000 & \\
\hline 18 & 8 & 17.8752 & \\
\hline 19 & 17 & 17.4577 & \\
\hline 20 & 20 & 18.1817 & \\
\hline 21 & 5 & 18.1522 & \\
\hline 22 & 1 & 18.3060 & \\
\hline 23 & 3 & 18.3060 & \\
\hline 24 & 24 & 18.0042 & \\
\hline
\end{tabular}

\section{A HEURISTIC APPROACH TO BLADE ARRANGEMENT}

In [1], a good heuristic for blade matching was obtained by first ordering the blades in $d$ and then matching neighbouring blade pairs or triples in the ordered list. This result, and the above discussion of the effect of blade pairing, suggests what turns out to be a fast and effective heuristic algorithm for blade arrangement. We assume that $N$ is even, so that there are $N / 2$ rotor diagonals each of which accommodates a pair of blades, and that the blades are ordered in descending order of $d$. The algorithm consists of the following steps:

1. Assign blades 1,2 (in the ordered list) to an arbitrary rotor diagonal (ie. to opposite rotor positions) which can be taken to form the positive and negative $x$ axes in an $x, y$ co-ordinate system.

2. Search for the best position pair for blades 3,4 from the remaining unoccupied rotor diagonals; for each diagonal, check whether the assignment should be 3,4 or 4,3 . The criterion for selection is the minimum of the sum of squared cumulative $x$ - and $y$ - components of $\boldsymbol{d}$ (see Equation (1)), cumulative meaning a sum over blades already positioned. Delete the position pair giving the "best" assignment from the list of unoccupied diagonals.

3. Repeat step 2 for successive blade pairs $\{(5,6), \ldots(N-1, N)\}$ until the assignment is complete.

This algorithm entails a number of computations bounded by a cubic polynomial in $N$, so that there will be no exponential (or worse) explosion of computing time with $N$.

For moderate $N(\leq 12)$, an optimal assignment of blades to rotor positions can be computed in a reasonable time by a total enumeration of all possible arrangements. We have 
Table 3: Comparison of Heuristic and Optimal Blade Assignments

\begin{tabular}{lccccc}
\hline & \multicolumn{2}{c}{ Normalised Eccentricity $\times \mathbf{1 0}^{\mathbf{3}}(\mathbf{m i n}, \mathbf{m e a n}, \mathbf{m a x})$} & & \multicolumn{2}{c}{ CPU secs } \\
\cline { 2 - 3 } & Heuristic & Optimal & & Heuristic & Optimal \\
$\mathbf{6}$ & $(0.246,0.987,2.584)$ & $(0.126,0.615,2.153)$ & & 0.0001 & 0.028 \\
$\mathbf{8}$ & $(0.075,0.552,1.516)$ & $(0.008,0.083,0.287)$ & & 0.0001 & 0.109 \\
$\mathbf{1 0}$ & $(0.037,0.361,1.120)$ & $(0.001,0.011,0.035)$ & & 0.0001 & 7.92 \\
$\mathbf{1 2}$ & $(0.043,0.355,0.710)$ & $(0.000,0.001,0.002)$ & & 0.0001 & 874.0 \\
$\mathbf{2 4}$ & 0.019 & - & & 0.0078 & -
\end{tabular}

programmed both the heuristic and the total enumeration in MATLAB. Table 3 shows the results of the comparison. For each value of $N \leq 12,20$ blade sets were randomly selected from the data in Table 2, ensuring that no duplication of blade sets occurred. For $N=24$, there is only one possible blade set, and a computation of the optimal assignment, which would have entailed a prohibitive computing time, was not attempted. The computing times are those to be expected on a reasonably fast current desktop PC.

The results show that for $\mathrm{N} \leq 10$, the computation of an optimal blade assignment by total enumeration entails negligible computing time, and would therefore be the best method to use. For larger values of $N$, our proposed heuristic gives good eccentricity values (compare the value for $N=24$ with Figures 3 and 4), although still at least two orders of magnitude beyond the optimal. Thus, if minimal rotor balance in a 24-blade rotor is an important consideration, and computing times of the order of 30 mins on a PC are considered feasible, one could proceed by arbitrarily splitting the blade set into two, computing the optimal assignment of 12 blades to each half-rotor, and then finding the best way of merging the two. For a 24-blade rotor, this should give near perfect balance.

\section{DISCUSSION AND CONCLUSIONS}

The minimising of the eccentricity of the rotor centre of mass by judicious arrangement of blades was considered for a large, multi-bladed rotor used on water pumping windmills. The number of blades in the rotor is also the number of blades manufactured in each production batch. This is almost the opposite problem to that for electricity-generating turbines where the blade pairs or triples are to be optimally matched to form pairs or triples from a large batch, Hitz \& Wood [1]. Their branch and bound algorithms are unsuitable in the present test case of 24 blades, because the contribution to the eccentricity from the individual blades can be negative for blade numbers greater than 4 . Even if this problem could be overcome, the number of possible blade arrangements is probably too great to allow an exact determination of the minimum eccentricity.

Two factors mitigate this problem. The first is that computation of minimum eccentricity by exact enumeration of all blade arrangements is practicable for approximately 12 blades or less. Second is the expectation that random blade selection will become a good strategy as the number of blades increases. This is shown dramatically in Figure 3 for the eccentricity of 10,000 random selections of a 12-blade and 24-blade rotor from the blades in Table 1 . The average eccentricity for the second case is significantly lower than for the first. A refinement to random selection is to first pair the blades by ordering and then randomly select the pairs. This reduces the average eccentricity in Figure 4 from that shown in Figure 3. A further significant reduction occurs when the every second blade pair is swapped before random selection. It appears that this strategy is effective as it changes the sign of the summand in the last sum of Equation (4). With this strategy it takes less than 15 seconds to generate 10,000 random selections on a moderately powerful laptop. It is almost certain that the selection giving the minimum eccentricity will be an excellent arrangement, as demonstrated in Table 2. 
The heuristic developed in Section 5 obtained eccentricities lower than the mean of those found by random selection in a fraction of the time required to generate and search 10,000 random arrangements. The eccentricities from the heuristic were significantly higher than the minimal values found by searching all possible arrangements for 12 blades or less, but are still well within the 'default' maximum eccentricity assumed in the IEC standard for the safety of small wind turbines, [1].

\section{ACKNOWLEDGMENTS}

The authors are grateful to Mike Harries of Kijito Windpower Ltd for permitting and facilitating the measurement of his blades and for permission to publish the results of our work.

\section{REFERENCES}

1. Hitz' K. L., Wood, D.H., On blade matching after batch production, Wind Engineering, to appear.

2. IEC 61400-2 Ed. 2.0 Design requirements for small wind turbines, 2006.

3. Harries, M.A.A. (2009). Case Study Of The Development in Kenya of the Third Generation Kijito Windpump, Wind Engineering 33, 2, pp 175-182. 
